\title{
A Time-dependent and Anisotropic Force Field Model For Galactic Cosmic Ray Flux
}

\author{
Grace D. Ihongo* \\ University of Aberdeen, $U K$ \\ E-mail: g.ihongoeabdn.ac.uk

\section{Charles H T. Wang} \\ University of Aberdeen \\ E-mail: c.wang@abdn.ac.uk
}

Galactic cosmic ray flux calculated at 1 au within the energy range (0.2-88) Ge V using the model is presented. The relationship between the calculated flux and solar wind is analyzed and presented. Short-time variation of the flux at a fixed energy range is also calculated, and this is used to predict galactic cosmic ray intensity variation at earth.

The 34th International Cosmic Ray Conference,

30 July- 6 August, 2015

The Hague, The Netherlands

\footnotetext{
* Speaker.
} 


\section{Introduction}

The time- dependent and anisotropic force field model is a model developed to model macroscopically, the transport of galactic cosmic rays in the heliosphere including its flux calculation and short time variation at earth (1AU).

This macroscopic modeling is based on the fact that galactic cosmic ray flux traveling through the heliosphere is influenced by the solar wind and the interaction processes are namely:

- Convection in the solar wind

- Drift Motion due to magnetic field irregularities

- Diffusion in the HMF

- Adiabatic cooling(change in momentum or energy).

The transport equation describing the above processes was first derived by Parker and this is written here in its simplest form as [1,2]

$$
\frac{\partial f(\boldsymbol{r}, p, t)}{\partial t}+\nabla \cdot \boldsymbol{S}-\underbrace{\frac{1}{3} \nabla \cdot \boldsymbol{V} \frac{\partial f(\boldsymbol{r}, p, t)}{\partial \ln p}}_{Q}=0
$$

where $\boldsymbol{S}=4 \pi p^{2}(\boldsymbol{C} \boldsymbol{V} f(\mathbf{r}, p, t)-\boldsymbol{\kappa} \cdot \nabla f(\mathbf{r}, p, t))$

Is the differential current density and $C=\frac{-1}{3} \frac{\partial \ln f}{\partial \ln p}$ Is the Compton-Getting factor, $p$ is the momentum and $Q$ is the term describing energy losses.

There has been various approximations to the Parker transport equation among which are [2] the diffusion convection model, the force model and the $1 D$-numerical solution however, both have not been able to model inclusively, the flux variation with time and the exact relationship between galactic cosmic ray flux and the solar wind.

Here we studied vigorously, the short time variation of galactic cosmic ray flux calculated at $1 A U$ using a time dependent and anisotropic force field model where, a variable decrease in flux is observed and the flux variation at earth is found to be dependent on the solar wind.

\section{The time-Dependent and Anisotropic Force Field Model Equation}

We implore the following assumptions to solve eqn (1.1):

- There is a quasi-Stationary state such that $\frac{\partial f}{\partial t}=0$ and no sources

- There are no energy losses such that $\nabla \cdot V=0$ for $r \geq 1 A U$

- Galactic cosmic rays are carried by the solar wind

- The solar wind is radially dependent 
- No particle drifts

- There is a small anisotropy in the solar wind such that $V=V(r, \theta, \tau)$

- The heliosphere is axially-symmetric in the heliocentric coordinate system where $\theta=\frac{\pi}{2}$

- Isotropic and parallel diffusion coefficient

- Perpendicular diffusion is negligible

- Earth's velocity influence is negligible compared to solar wind speed.

The solution is a new transport equation known as the time-dependent and anisotropic force field model. This is written as

$$
\frac{\partial f(r, \theta, p, \tau)}{\partial r}+\frac{P V(r, \theta, \tau)}{3 \kappa} \frac{\partial f(r, \theta, p, \tau)}{\partial P}=0
$$

Where $V$ is the solar wind speed, $\kappa$ is the diffusion coefficient, $p$ is the momentum $\tau$ is the solar wind time which is transformed from the normal time $t$ to the solar wind frame (Note that detailed transformation equations are omitted here due to the restricted length of the paper).

Eqn 2.1 has the following analytical solution

$$
f(r, \theta, p, \tau)=F(\theta, \underbrace{-\int \frac{V(r, \theta, \tau)}{\kappa_{1}(r)} \mathrm{d} r+3 \int \frac{\beta \kappa_{2}(p)}{p} \mathrm{~d} p}_{X})
$$

where $F$ is an arbitrary function to be fixed using the boundary values and $X$ is assumed to be a constant such that the general solution is

$$
f(r, p, \theta, \tau)=F(\theta, X) \equiv f(R, \theta, \tau)
$$

The general solution is explained in fig 1 . This yields the following specific solution

$$
\int_{p}^{p^{*}} \frac{\beta \kappa_{2}\left(p^{\prime}\right)}{p^{\prime}} \mathrm{d} p^{\prime}=\int_{r}^{R} \frac{V(r, \theta, \tau)}{3 \kappa_{0}} \mathrm{~d} r \equiv \phi(r, \theta, \tau)
$$

which yields

$$
\phi(r, \theta, \tau)=\int_{r}^{R} \frac{V(r, \theta, \tau)}{3 \kappa_{0}} \mathrm{~d} r
$$


The above solution is re-written in terms of cosmic ray flux and its kinetic energy using the following relation $[3,4]$ :

$$
J(p)=p^{2} f(p)
$$

where

$J(p)$ is the observed cosmic ray flux spectrum, $p$ is the rigidity of cosmic ray particles and $f$ is the omnidirectional distribution function of cosmic ray intensity.

Here, we convert the above spectrum to kinetic using the relation:

$$
\left(\frac{A}{Z}\right) \sqrt{T\left(T+2 T_{0}\right)}
$$

Where $A$ and $Z$ are the mass and atomic numbers of protons respectively.

This yields the model solution in terms of flux and kinetic energy as

$$
j(r, \theta, T)=j_{T^{*}}\left(\frac{T\left(T+2 T_{0}\right)}{T\left(T+2 T_{0}\right)+2\left(\sqrt{T\left(T+2 T_{0}\right)}\right) \Phi(r, \theta, \tau)+\Phi^{2}(r, \theta, \tau)}\right)
$$

where $\Phi=\frac{Z e \phi(r, \theta, \tau)}{A}$ and $\phi(r, \theta, \tau)=\frac{V(r, \theta, \tau)}{3 \kappa_{0}}(R-r), A$ is the atomic mass, $Z$ is the atomic number of cosmic rays protons and $e$ is the electronic charge $. J_{T^{*}}\left(R, T^{*}\right)=b \beta\left(\frac{A}{Z e}\left(\sqrt{T^{*}\left(T^{*}+2 T_{0}\right)}\right)^{-a}\right.$

where $b$ and $a$ are constants and $\beta=1$.

$T$ is the kinetic energy and $T_{0}$ is the rest mass of a proton.

$*$ is used to denote the values at the boundary of the heliosphere. 
Figure 1: Representation of the general analytical solution(egn 2.3): The blue dashes is the inner heliosphere, the black is the outer heliosphere, $r, R, \tau$ are the inner heliospheric distance, outer heliospheric distance and the time transformed to the solar wind frame respectively. $X, Y, Z$ represents the heliocentric coordinate system where $\theta=\frac{\pi}{2}$

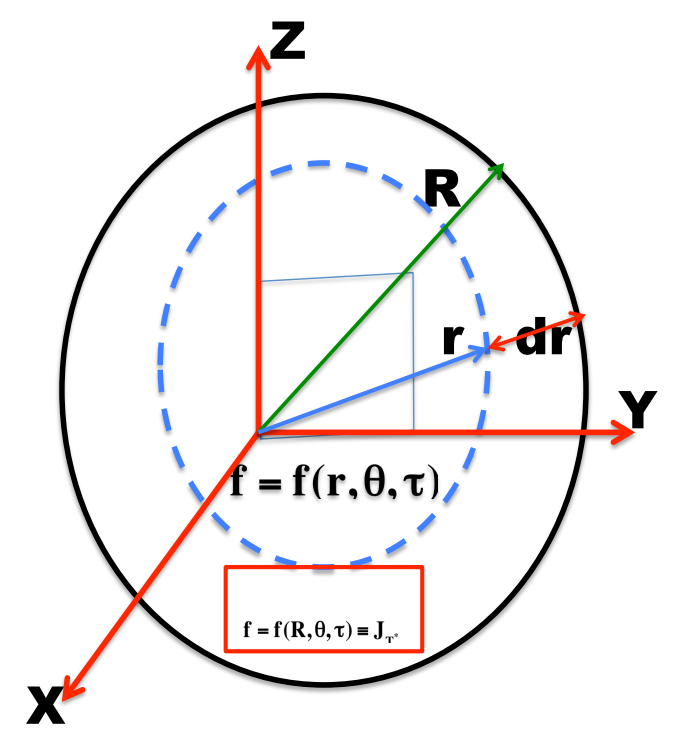

The idea in fig 1 is that, a solution $f(R, \theta, \tau)$ is first obtained at the boundary and then used to obtained the solution $f(r, \theta, \tau)$ inside the heliosphere. We assume that particles travels only along the $Z$-direction such that solar wind expansion is only radially dependent and both $\theta$ and $\phi$ are neglected. 


\section{Results}

Figure 2: Calculation of flux variation using the time-dependent and anisotropic force field model at fixed energy ranges as seen on the color bar left top panel, bottom left is solar wind variation with time. The right panel is model comparison with neutron monitor count rates; top left is this model, bottom left are counts are from Moscow NM: cro.izmiran.rssi.ru/mosc/main.htm and Oulu NM: cosmicrays.oulu.fi respectively).. The fit is done for: $T_{0}=0.9384 G e V, R=100 A U, r=1 A U, \theta=\frac{\pi}{2}, a=2.788, b=1.82 e 4, \kappa=$ $7.3 \times 10^{20} \beta \mathrm{Pcm}^{2}(\mathrm{GV}) / \mathrm{s}$. Solar wind data are from ACE website: srl.caltech.edu/ACE/ASC/rtsw.html, and the black line is the local interstellar or the boundary spectrum. Note that the boundary spectrum is multiplied by a correction factor of $10^{2}$ to correct its uncertainties.
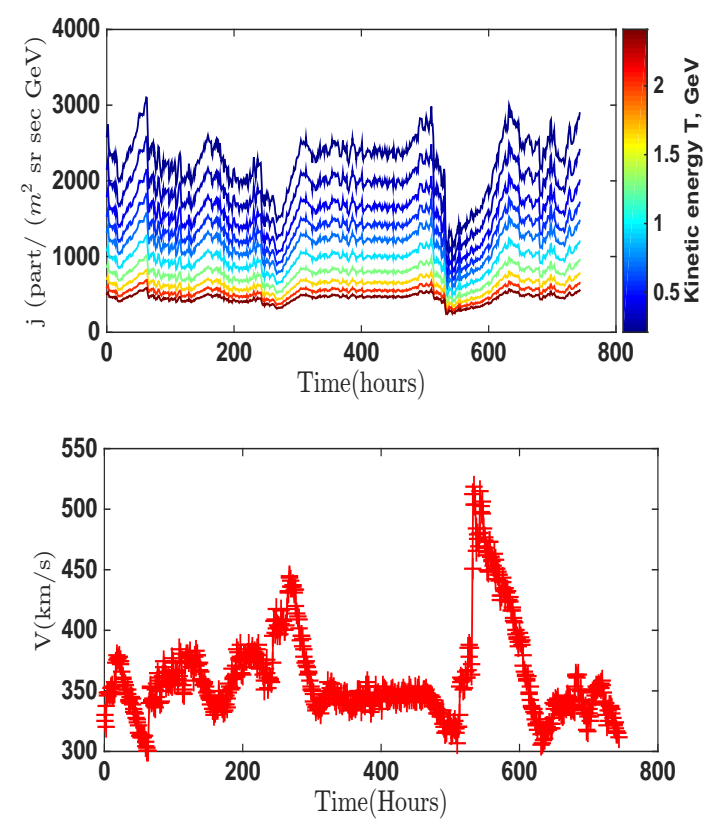
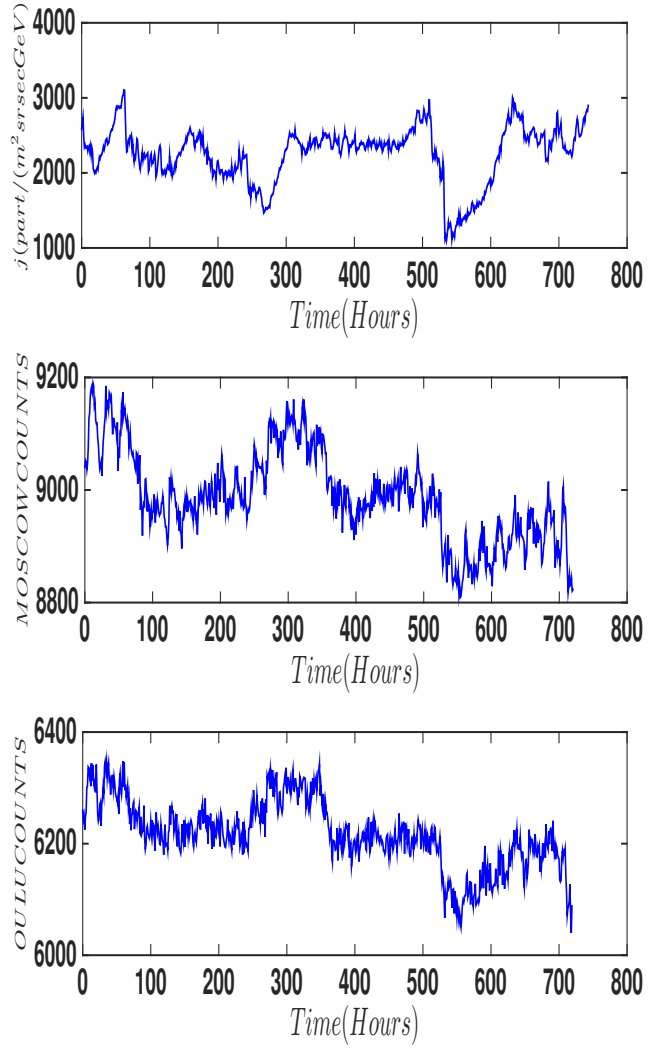


\section{Discussion}

As seen in fig 2; galactic cosmic ray flux varies with time and the variable decrease in flux is due to solar wind variation. The flux variation and solar wind are anti-correlated.

The sharp decrease in flux at $270 \mathrm{hrs}$ and between $520-550 \mathrm{hrs}$ are due to sudden increase in solar wind and we describe this effect as solar wind related Forbush decrease while the sharp increase in flux at $300 \mathrm{hrs}$ indicate a solar wind related Forbush increase. This means that solar wind is a possible cause of Forbush decreases usually observed on galactic cosmic rays in addition to the well known case of corona mass ejections [5]. It would be recalled that corona mass ejections has been named the cause of Forbush decreases [5].

The right top panel in fig 2 is this model while the last bottom panels are count rates from the MOSCOW and OULU cosmic ray stations respectively, this is done to compare the model with observational data from the cosmic ray stations within the same time frame to see if our model agrees with data and it can be seen clearly that the model is consistent with data as the same variable decrease in flux is observed with data within this time frame.

\section{Summary and Progress}

Galactic cosmic ray flux has been modeled by solving the Parker cosmic ray transport equation using reasonable assumptions. A variable decrease in flux caused by the anisotropy in the solar wind is observed and the solar wind has been identified a possible cause of Forbush decreases or increases observed on galactic cosmic ray flux in addition to corona mass ejections.

We intend to use our result to predict galactic cosmic rays flux variation on earth. 


\section{Acknowledgements}

We sincerely appreciate the following people /groups for their useful contributions.

- Members of the QG2 University of Aberdeen, UK.

- H. Moraal, School of Physics, Potchefstroom University, Potchefstroom, 2520, South Africa.

- R. A. Caballero-Lopez, Institute for Physical Science and Technology, University of Maryland, College Park, MD 20742, USA

\section{References}

[1] Parker, E. N. (1965), The passage of energetic particles through inter- planetary space, Planet. Space Sci., 13, 9.

[2] Caballero-Lopez R.A. and Moraal H. 2004, Limitations of the force equation to describe cosmic ray modulation Journal of Astrophysical Res. vol 109,A01101, doi10.1029/2003JA010098

[3] Moraal.H (2013) Cosmic-Ray Modulation Equations Space Sci Rev 176 : 299 - 319.

[4] McDonald F.B. Caballero-Lopez R.A. and Moraal H. (2005) Termination shock and heliosheath effects on cosmic ray energy changes in the heliosphere 29th International Cosmic Ray Conference Pune 00, 101 ÂUU104.

[5] Naglaa E. Aly (2013) The characteristics of Forbush decrease of cosmic rays intensity IJRRAS 15 (3) 